\title{
A 10-Year History of Anti-Smoking Campaigns and Enlightenment Activities for Chronic Obstructive Pulmonary Disease for Citizens at the Plaza in Ebina City
}

\author{
Yozo Sato ${ }^{1,2}$, Namiko Taniuchi ${ }^{1,2}$, Shota Kaburaki ${ }^{1,2}$, Naohiro Aruga ${ }^{1}$, \\ Kaoru Kubota ${ }^{2}$, Masahiro Seike ${ }^{2}$, Yoshimitsu Yamano ${ }^{1,2}$ and Akihiko Gemma ${ }^{2}$ \\ ${ }^{1}$ Department of Respiratory Medicine, Ebina General Hospital, Kanagawa, Japan \\ ${ }^{2}$ Division of Pulmonary Medicine and Oncology, Graduate School of Medicine, Nippon Medical School, Tokyo, Japan
}

Background: The prevalence of chronic obstructive pulmonary disease (COPD) is $8.6 \%$ in Japan and $10 \%$ worldwide. Unfortunately, many patients with COPD are not correctly identified and appropriately educated regarding the condition. In this paper, we demonstrate that some citizens of Ebina City with symptoms suspicious for COPD, such as cough, sputum production, and shortness of breath, have undiagnosed COPD. We describe our activities to raise awareness of COPD through a 10-year campaign.

Methods: From 2006 to 2015, we developed activities to raise awareness of COPD, including public lectures, utilization of pulmonary function tests, and questionnaires on subjective symptoms and knowledge of COPD.

Results: Among 1,206 participants aged $>40$ years, COPD was suspected in $5.6 \%$, as indicated by airway obstruction (i.e. forced expiratory volume in 1 second (FEV1)/forced vital capacity ratio $(\mathrm{FVC})<0.70$ ). However, most of these participants were not diagnosed with COPD. Furthermore, half of these participants had not consulted a medical institution despite demonstrating symptoms. Results of the COPD awareness questionnaire, which was administered to 1,055 people, indicated that $65 \%$ of survey respondents were unaware of COPD.

Conclusions: There are individuals with symptoms suspicious for COPD who are unaware of the disease at the Plaza in Ebina City. Clinicians have a responsibility to raise public awareness of COPD and to reduce the prevalence of COPD and its associated mortality. (J Nippon Med Sch 2019; 86: 32-37)

Key words: anti-smoking campaign, chronic obstructive pulmonary disease, disease awareness activity

\section{Introduction}

Chronic obstructive pulmonary disease (COPD) is an inflammatory disease of the lungs caused by long-term inhalation exposure to harmful substances, mainly tobacco smoke. Patients with COPD exhibit progressively worsening airflow obstruction. COPD is diagnosed by a ratio of forced expiratory volume in 1 second (FEV1) to forced vital capacity (FVC) of less than 0.70 when measured after administration of an inhaled bronchodilator. Before diagnosing COPD, other diseases causing airflow obstruction must be ruled out ${ }^{1}$. According to the statistics of the Ministry of Health, Labor and Welfare, the number of deaths attributable to COPD has increased and $>15,000$ people die of COPD in Japan every year'. In men and women, COPD is the eighth and tenth leading cause of death, respectively. In addition, according to the World Health Organization (WHO) ${ }^{3}$, COPD claimed 3.2 million lives and was the fourth leading cause of death worldwide in 2015.

The Nippon COPD Epidemiology (NICE) study, which is a large-scale epidemiologic COPD survey in Japan, showed that $8.6 \%$ of citizens aged $>40$ years (approximately 5.3 million people) suffered from COPD ${ }^{4}$. According to the Ministry of Health, Labor and Welfare's statistics, the number of patients diagnosed with COPD in hospitals in 2014 was 260,000 nationwide ${ }^{3}$. Mild subjec-

Correspondence to Namiko Taniuchi, Division of Pulmonary Medicine and Oncology, Graduate School of Medicine, Nippon Medical School, 1-1-5 Sendagi, Bunkyo-ku, Tokyo 113-8603, Japan

E-mail: taniuchi@nms.ac.jp

Journal Website (http://www2.nms.ac.jp/jnms/) 
Table 1 Participant questionnaire

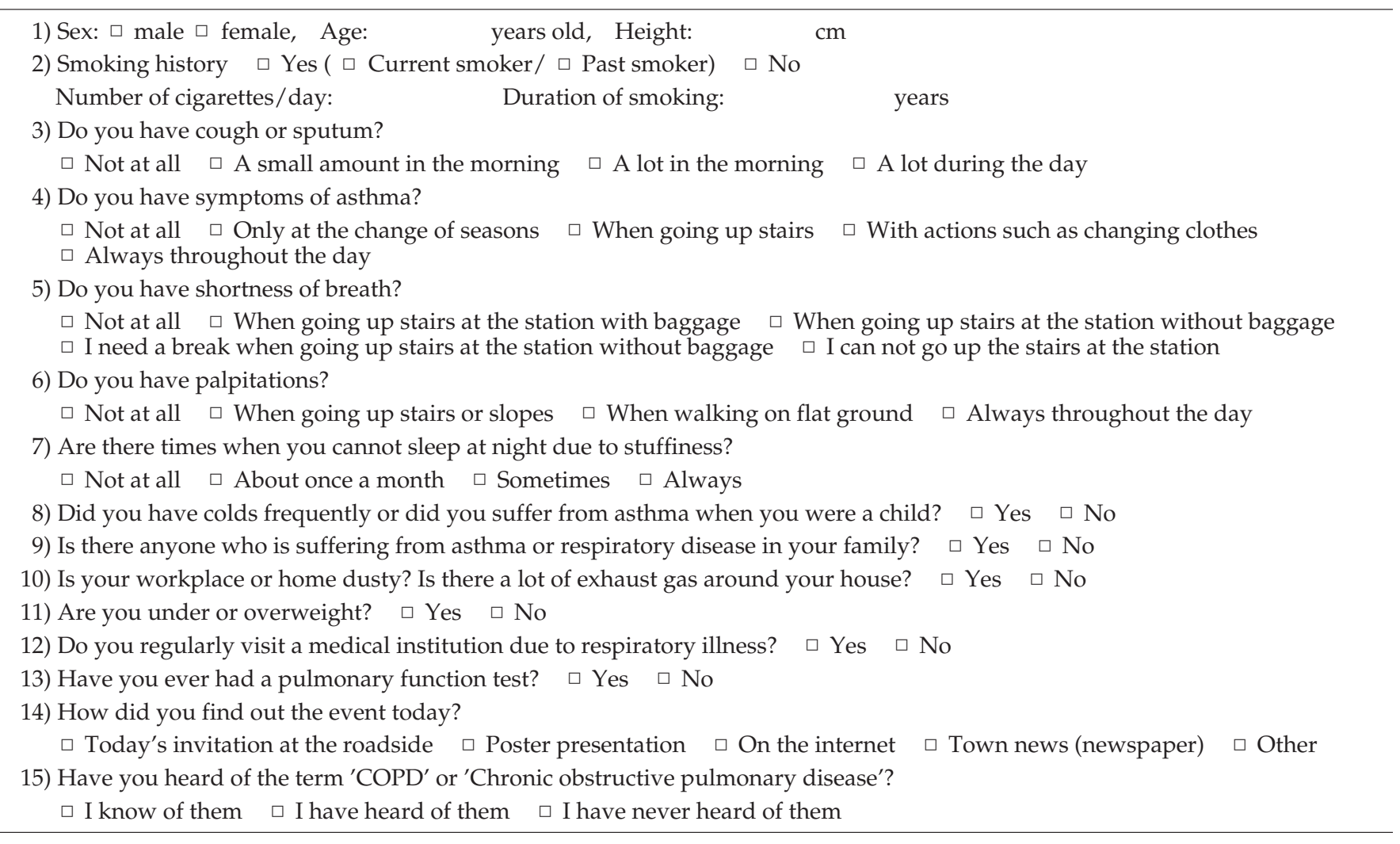

tive symptoms, older age, and mild spirometry findings are reasons for undiagnosed COPD. Low awareness of COPD is an additional important factor. In the December 2016 internet survey of the Global Initiative for Chronic Obstructive Lung Disease (GOLD) Committee in Japan, COPD awareness was only $25 \%{ }^{5}$. In addition, two studies conducted in Spain ${ }^{6,7}$ respectively found that only $17.0 \%$ and $8.6 \%$ of symptomatic individuals in the general population recognize the term "COPD".

In this survey, we clarified the situation that citizens with symptoms suspicious for COPD, such as cough, sputum, and shortness of breath, exist in a state of undiagnosed COPD and that COPD itself is not acknowledged by citizens in Ebina City. We also raised awareness of COPD through 10-year activities and increased recognition rate.

\section{Materials and Methods}

From 2006 to 2015, we recruited participants from the general population at the Plaza in Ebina City, Kanagawa Prefecture. Surveys were completed during the autumnal holiday every year in line with World COPD Day and were conducted by respiratory medicine doctors, clinical examination technicians, and nurses from Ebina General Hospital and general volunteers. The study was spon- sored by Ebina City Medical Association, Japan Boehringer Ingelheim Co., Ltd., and Chest Corporation. Participants completed questionnaires on variables, such as age, sex, height, smoking history, presence or absence of respiratory symptoms, and knowledge of COPD (Table 1). Participants received basic instruction on COPD using slides and pamphlets. Then, participants underwent pulmonary function testing (PFT) (CHEST GRAPH Junior HI-101 from Chest Corporation). Finally, participants received feedback from respiratory medicine doctors regarding their PFT results, COPD, and smokinginduced health damage.

We analyzed the PFTs of 1,623 participants, including results of 10-year studies, and extracted participants with suspected COPD (FEV1/FVC ratio $<0.70$, or $\mathrm{FEV} 1 \%<$ $70 \%$, and current or existing smokers), and excluded participants with suspected asthma from the questionnaire responses. Participants who answered "once a month", "sometimes", or "always" for question 7 or who answered "Yes" for questions 8, 9, or 12 were excluded as having asthma (Table 1). Those who did not answer "Not at all" on questions 3 or 5 were classified as having COPD symptoms (Table $\mathbf{1}$ ).

Airflow limitation and degree classification were determined per the diagnostic criteria of the GOLD $2018^{1}$. 


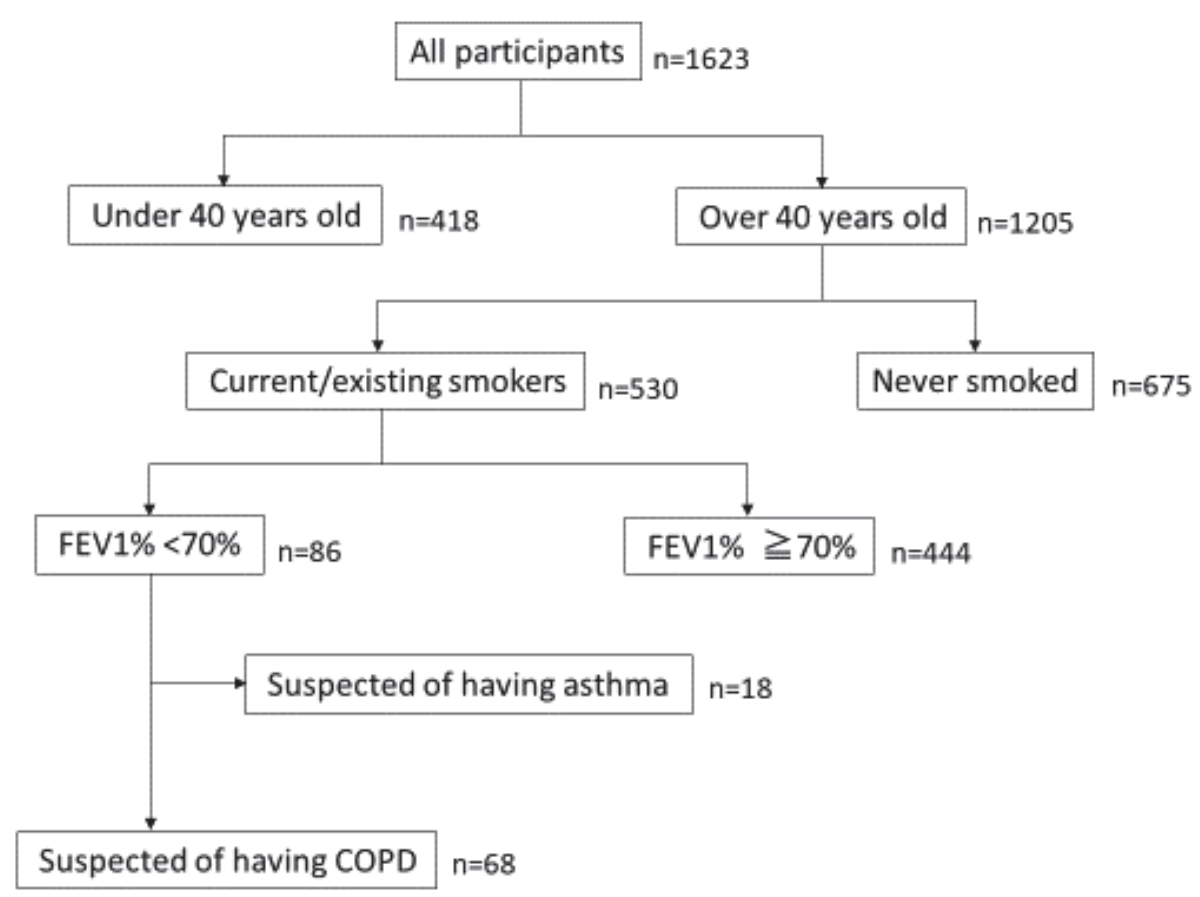

Fig. 1 Flow diagram of the study population. We divided 1,623 participants into those aged $>40(1,205)$ and $<40$ years $(418)$. Among them, 530 were current or existing smokers ( 86 had a forced expiratory volume in 1 second (FEV1)/forced vital capacity (FVC) ratio $<70 \%)$. Furthermore, after excluding those with suspected asthma, 68 (5.6\%) were suspicious for having chronic obstructive pulmonary disease.

Bronchodilators were not administered before PFT.

We used questionnaire responses to "Have you had a pulmonary function test?" and "Do you know the meaning of COPD?". A total of 1,055 participants, including those $<40$ years old, participated in the study from 2010 to 2015. Participants provided informed consent to publicize data obtained during this activity orally and via posters. The study was approved by the Ethics Committee of Ebina General Hospital.

\section{Results}

Figure 1 is a flow diagram of the study population for extracting participants suspicious for having COPD. Of 1,205 participants aged $>40$ years, 530 were current or existing smokers (86 had an FEV1\%<70\%) and 675 had never smoked. Furthermore, after excluding participants with suspected asthma, 68 (5.6\%) were suspicious for having COPD.

Among the total 1,205 participants aged $>40$ years, 148 $(12 \%)$ produced an FEV1\%<70\% (Table 2 ). Their average age was 62.7 years, which was roughly the same in each year. Proportionally, there were more women participants, so the results may reflect women's health awareness more than that of men. Over time, our COPD awareness efforts were recognized in the area, and the number of participants increased. The characteristics of participants aged $>40$ years are shown in Table 3 . There were 240 participants who had symptoms suspicious for COPD and were excluded from having asthma.

Figure 2 shows the relationship between COPD participants and symptoms. Among 240 participants with symptoms of COPD who were current or existing smokers, $38(15.8 \%)$ had an FEV1\%<70\%. Among 86 current or existing smokers with an FEV1\%<70\%, 38 (44.2\%) had symptoms of COPD and were excluded from having asthma. Also, 30 (34.9\%) participants had no symptoms of COPD.

Figure 3 shows the COPD awareness results from the questionnaire in 1,055 participants from 2,010 to 2,015. Only $15.0 \%$ of the participants participated in PFTs. A minority of participants knew $(12 \%)$ or heard $(22.8 \%)$ about COPD, whereas $65.2 \%$ did not know about COPD. These results indicated that COPD was poorly recognized by citizens. Among 68 participants suspicious for having COPD, 23 (33.8\%) recognized the term COPD. This recognition rate was as low as in the general population.

\section{Discussion}

In our survey, $5.6 \%$ of participants aged $>40$ years exhib- 
Antismoking Campaigns and Enlightenment Activities for COPD in Ebina City

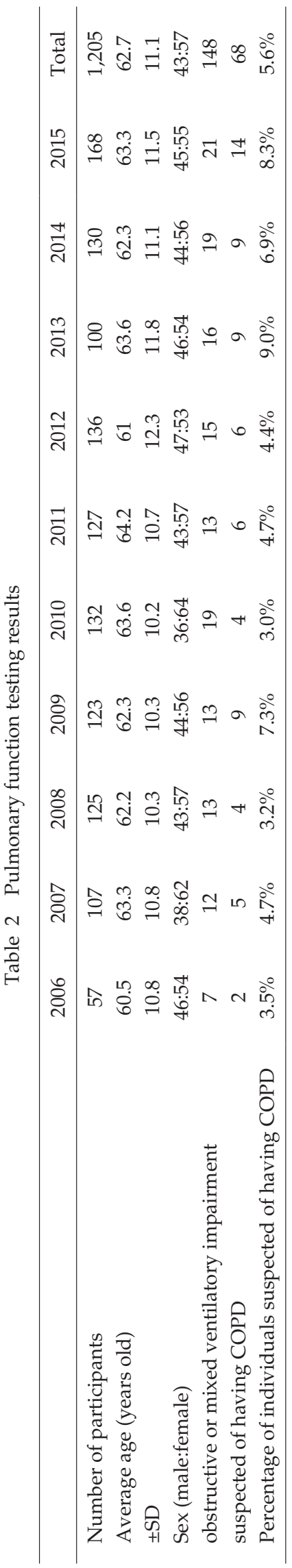

Table 3 Characteristics (1,205 participants who were over 40 years old)

\begin{tabular}{lc}
\hline \multicolumn{1}{c}{ Characteristics } & $\mathrm{n}$ \\
\hline Sex & \\
Female & 684 \\
Male & 521 \\
Smoking status & \\
Current/existing & 530 \\
Never & 675 \\
Symptoms suspected of COPD & 330 \\
Symptoms suspected of COPD and Exclude from & 240 \\
having asthma & \\
FEV1\%<70\% & 148 \\
FEV1\%<70\% and current/existing smoker & 86 \\
Suspected of having COPD & 68 \\
\hline
\end{tabular}

ited suspected COPD with an FEV $1 \%<70 \%$. The prevalence in our survey was lower than the $8.6 \%$ identified in the NICE study conducted in Japan ${ }^{4}$, and the approximate $10 \%$ prevalence in the PLANTINO $^{8}$ and BOLD $^{9}$ studies. The reason for this finding may be the high proportion of women in this study.

Several epidemiologic studies on COPD have been reported previously. A survey of the Takahata Town in Yamagata Prefecture revealed that $10.6 \%$ of respondents aged $>40$ years exhibited suspected COPD, as determined by spirometry results during health examinations ${ }^{10}$. According to a survey administered via primary care physicians in Chiba prefecture, $14 \%$ of patients exhibited suspected $\mathrm{COPD}^{11}$. Although a roughly $10 \%$ prevalence of COPD has been reported, differences were thought to be recognized depending on the target group.

Of note in our survey, $34.9 \%$ of the participants suspicious for having COPD had no symptoms of cough, sputum, and shortness of breath. A prospective study in Denmark has shown that the risk of exacerbation and pneumonia is high even in patients with asymptomatic $\mathrm{COPD}^{12}$. Therefore, it was thought necessary to detect as many patients as possible with asymptomatic COPD.

Over $95 \%$ of individuals with COPD in Japan are undiagnosed because of slow progression of COPD; therefore, many patients fail to recognize COPD symptoms until they become severe ${ }^{13}$. Second, medical professionals exhibit a poor ability to recognize $\mathrm{COPD}^{14}$. Finally, spirometry is underused ${ }^{15}$. Low COPD recognition is a major problem. Our questionnaire survey of 1,055 people revealed that only $34.8 \%$ recognized symptoms of COPD. Also, similar low recognition rates $(33.8 \%)$ were confirmed in participants suspicious for having COPD. The recognition rate was reported as only $17.0 \%$ and $8.6 \%$ in 


\section{Current/existing smokers and having COPD symptoms $(n=240)$}

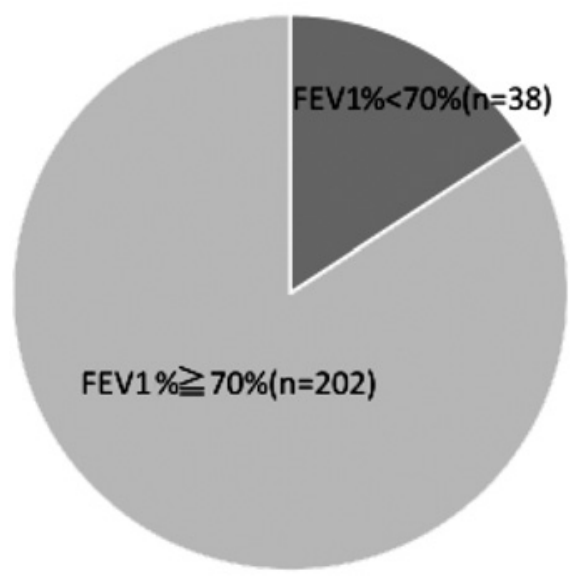

\section{Current/existing smokers and $\mathrm{FEV} 1 \%<70 \%(\mathrm{n}=86)$}

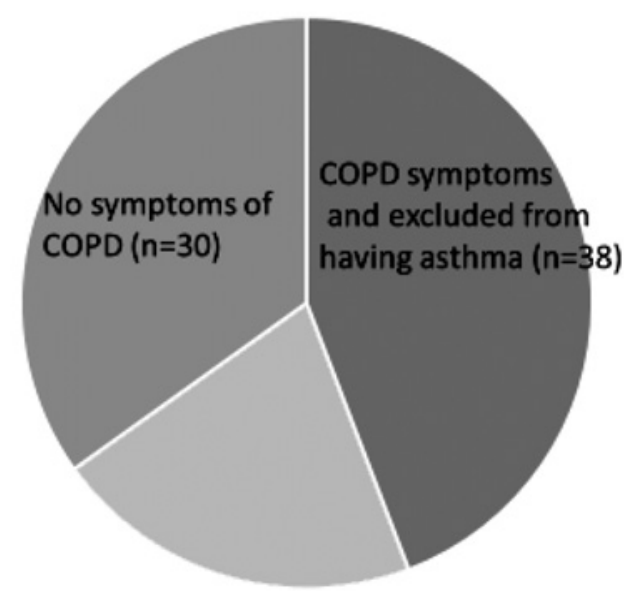

Fig. 2 Relationship between participants suspicious for having chronic obstructive pulmonary disease (COPD) and COPD symptoms. Among 240 participants with COPD symptoms who were current or existing smokers, $38(15.8 \%)$ had a forced expiratory volume in 1 second (FEV1)/forced vital capacity (FVC) ratio $<70 \%$. Among 86 current or existing smokers with an FEV1<70\%, $38(44.2 \%)$ had symptoms of COPD and were excluded from having asthma.

\section{5 participants from 2010 to 2015}

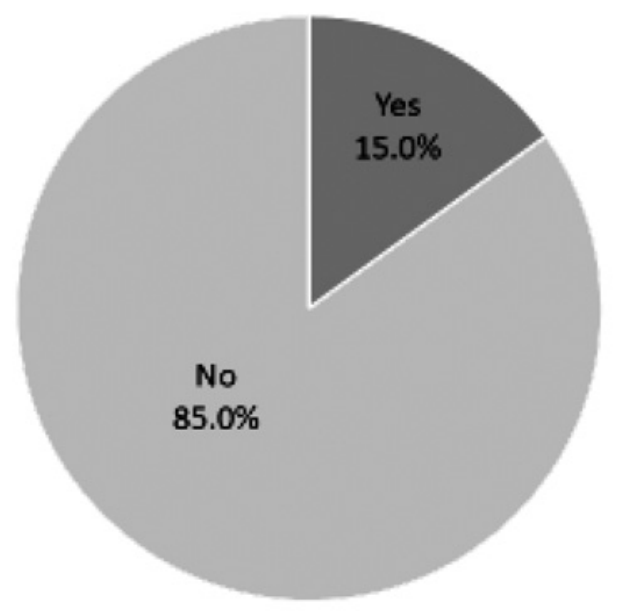

\section{Do you have undergone pulmonary} function test?

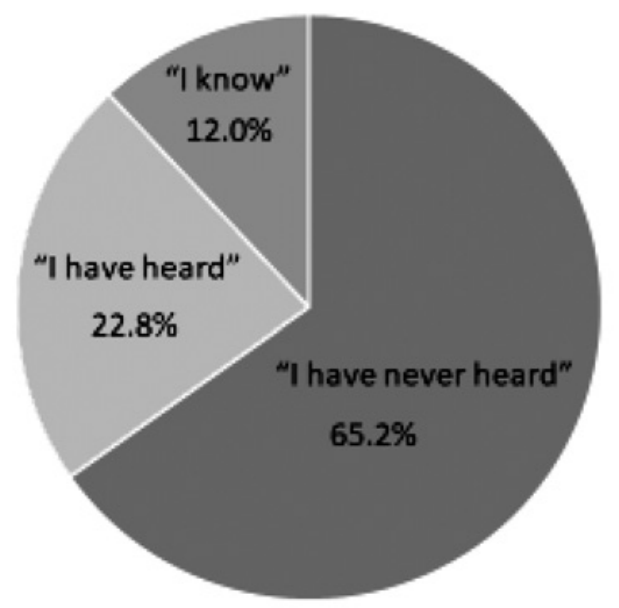

Do you know the word "COPD" or "Chronic obstructive pulmonary disease" ?

Fig. 3 Questionnaire results. We asked 1,055 participants whether they had undergone pulmonary function testing (PFT) and whether they knew the terms "COPD" or "chronic obstructive pulmonary disease" from 2010 to 2015. Only 15.0\% of the participants said that they had undergone a PFT or examination of lung age. Participants who answered "I have never heard of COPD" accounted for $65.2 \%$ of the total sample.

two studies conducted in Spain $^{6,7}$, and $8 \%$ in a study in France $^{16}$. A low recognition level leads to delays in diagnosis and an increase in mortality. COPD was added to "Health Japan 21" because of the magnitude of the loss of life and the medical economic loss, but also because it is "a disease that can be prevented and treated ${ }^{5} . "$
There are three limitations of this study: (1) participation in this study was not mandatory and was arbitrary, and there were more opportunities for health-conscious people; (2) study participants were anonymous and there is a possibility that the same person participated every year; and (3) diagnosis of COPD was uncertain because 
the PFT was not performed after bronchodilator administration in this study. Also, we did not perform chest auscultation, blood collection, or chest radiography, and exclusion of other diseases was not certain.

In our daily practice, clinicians must screen with questionnaires and spirometry to identify patients with latent COPD symptoms and promote early diagnosis and smoking cessation. We are hopeful that an $80 \%$ recognition rate can be achieved by 2022 by increasing the frequency of nationwide awareness activities.

\section{Conclusion}

In this survey, among participants aged $>40$ years, 5.6\% were suspicious for having COPD. It became clear that the number of patients with undiagnosed COPD is large. Also, only $35 \%$ of the population recognized the term COPD. Clinicians have a duty to raise the recognition rate of COPD through COPD awareness campaigns and to reduce COPD prevalence and mortality.

Conflict of Interest: The authors were sponsored by Ebina City Medical Association and Japan Boehringer Ingelheim Co., Ltd.

\section{References}

1. GLOBAL STRATEGY FOR THE DIAGNOSIS, MANAGEMENT, AND PREVENTION OF CHRONIC OBSTRUCTIVE PULMONARY DISEASE (2018 REPORT) (https://g oldcopd.org/wp-content/uploads/2017/11/GOLD-2018-v 6.0-FINAL-revised-20-Nov_WMS.pdf).

2. Ministry of Health, Labor and Welfare: Overview of population dynamics statistics: Heisei 20 years.

3. World Health Organization; 2017. http://www.who.int/ mediacentre/factsheets/fs310/en/. Accessed April 13, 2018.

4. Fukuchi $Y$, Nishimura $M$, Ichinose $M$, Adachi M, Nagai A, Kuriyama T, Takahashi K, Nishimura K, Ishioka S, Aizawa $\mathrm{H}$, Zaher C: COPD in Japan: the Nippon COPD Epidemiology study. Respirology 2004; 9: 458-465.

5. Shoji K: Epidemiology of COPD and Preventive Health Japan 21 (second) mainly. The Journal of the Japanese Society of Internal Medicine 2015; 104: 1059-1066.

6. Soriano JB, Calle $\mathrm{M}$, Montemayor $\mathrm{T}$, Alvarez-Sala JL, Ruiz-Manzano J, Miravitlles M: The general public's knowledge of chronic obstructive pulmonary disease and its determinants: current situation and recent changes. Arch Bronconeumol 2012; 48: 308-315.
7. Miravitlles M, de la Roza C, Morera J, Montemayor T, Gobartt E, Martín A, Alvarez-Sala JL: Chronic respiratory symptoms, spirometry and knowledge of COPD among general population. Respir Med 2006; 100: 1973-1980.

8. Menezes AM, Perez-Padilla R, Jardim JR, Muino A, Lopez MV, Valdivia G, Montes de Oca M, Talamo C, Hallal PC, Victora CG; PLATINO Team: Chronic obstructive pulmonary disease in five Latin American cities (the PLATINO study): a prevalence study. Lancet 2005; 366: 1875-1881.

9. Buist AS, McBurnie MA, Vollmer WM, Gillespie S, Burney $\mathrm{P}$, Mannino DM, Menezes AM, Sullivan SD, Lee TA, Weiss KB, Jensen RL, Marks GB, Gulsvik A, Nizankowska-Mogilnicka E; BOLD Collaborative Research Group: International variation in the prevalence of COPD (the BOLD Study): a population-based prevalence study. Lancet 2007; 370: 741-750.

10. Osaka D, Shibata $Y$, Abe S, Inoue S, Tokairin $Y$, Igarashi A, Yamauchi K, Kimura T, Sato M, Kishi H, Takabatake $\mathrm{N}$, Sata M, Watanabe T, Konta T, Kawata S, Kato T, Kubota I: Relationship between habit of cigarette smoking and airflow limitation in healthy Japanese individuals: the Takahata study. Intern Med 2010; 49: 1489-1499.

11. Nakamura M, Sakurai T, Tatsumi K: Early Detection of COPD in Patients with Dyspnea on Exertion in General Particle. Japanese Journal of Clinical Physiology 2010; 40: 199-205.

12. Colak Y, Afzal S, Nordestgaard BG, Vestbo J, Lange P: Prognosis of asymptomatic and symptomatic, undiagnosed COPD in the general population in Denmark: a prospective cohort study. Lancet Respir Med 2017; 5: 426434.

13. Kornman O, Beeh KM, Beier J, Geis UP Ksoll, Buhl R: Newly diagnosed chronic obstructive pulmonary disease. Clinical features and distribution of the novel stages of the Global Initiative for Obstructive Lung Disease. Respiration 2003; 70: 67-75.

14. Kamimura T, Oshita Y, Koga T, Sueyasu Y, Kinoshita M, Aizawa H: Under diagnosis of COPD in hospitalized patients. Eur Respir J 2002; 20: 122s.

15. Rennard S, Decramer M, Caiverley PM, Pride NB, Soriano JB, Vermeire PA, Vestbo J: Impact of COPD in North America and Europe in 2000: subjects' perspective of Confronting COPD International Survey. Eur Respir J 2002; 20: 799-805.

16. Roche N, Perez T, Neukirch F, Carre P, Terrioux P, Pouchain D, Ostinelli J, Suret C, Meleze S, Huchon G: High prevalence of COPD symptoms in the general population contrasting with low awareness of the disease. Rev Mal Respir 2011; 28: e58-e65.

(Received, February 13, 2018)

(Accepted, November 8, 2018) 